\title{
Chest wall reconstruction with perforator flaps after wide full-thickness resection
}

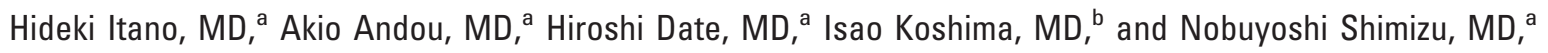

Okayama, Japan

$\mathrm{P}$ ioneering work by Koshima and colleagues ${ }^{1}$ and Kroll and Rosenfield ${ }^{2}$ in the late 1980s introduced perforator flaps, a new type of surgical flap based on musculocutaneous perforator arteries with exclusion of the passive muscle carrier. Perforator flaps combine the reliable blood supply of musculocutaneous flaps with the reduced donor site morbidity of a skin flap. We report the successful reconstruction of a wide chest wall defect using perforator flaps.

\section{Clinical Summary}

A 68-year-old man had a progressively enlarging left infrascapular desmoid tumor associated with left shoulder pain over the past 12 months. A hard, multinodular mass measuring $10 \times 11 \times 5 \mathrm{~cm}$ was noted on his left back, and invasion to the ribs, latissimus dorsi muscle, and skin was demonstrated. Surgical intervention was performed on September 22, 2000. The chest wall resection with the 3-cm surgical margin included parts of the sixth, seventh, eighth, and ninth ribs and the chest wall muscles and skin. This resulted in a full-thickness chest wall defect of $13.0 \times 16.5 \mathrm{~cm}$. A tension-applied double Marlex mesh was used for the skeletal reconstruction of the chest wall. After confirming the course of the perforator vessels by using Doppler ultrasound flowmetry, 2 pedicled perforator flaps were outlined adjacent to the defect. These flaps were large enough to cover the defect area and were based on the horizontal cutaneous branches of circumflex scapular vessels and the intercostal, thoracodorsal musculocutaneous perforators. ${ }^{3}$ These 2 flaps were then carefully dissected and elevated from the underlying muscle, with the perforator vessels visualized at the flap base to confirm proper flap orientation. The flaps were then rotated and transposed to cover the defect in an airtight fashion. The donor site was skin grafted with a split-thickness meshed skin graft harvested from the patient's left hip (Figures 1 and 2). No flail chest was encountered postoperatively. Weight bearing onto the flaps and left shoulder movement were prohibited for 2 weeks postoperatively. The wound remained airtight, without infection, flap necrosis, or dehiscence. Histopathologic diagnosis was com-

From the Departments of Cancer and Thoracic Surgery ${ }^{\mathrm{a}}$ and Plastic and Reconstructive Surgery, ${ }^{\mathrm{b}}$ Okayama University Graduate School of Medicine and Dentistry, Okayama, Japan,

Received for publication March 3, 2006; accepted for publication March 15, 2006

Address for reprints: Hideki Itano, MD, Section of Thoracic Surgery, Kure Kyosai Hospital, 2-3-28 Nishi-chuo, Kure City, Hiroshima 737-8505, Japan (E-mail: itano_h@ybb.ne.jp).

J Thorac Cardiovasc Surg 2006;132:e13-4

$0022-5223 / \$ 32.00$

Copyright $\odot 2006$ by The American Association for Thoracic Surgery doi:10.1016/j.jtcvs.2006.03.024
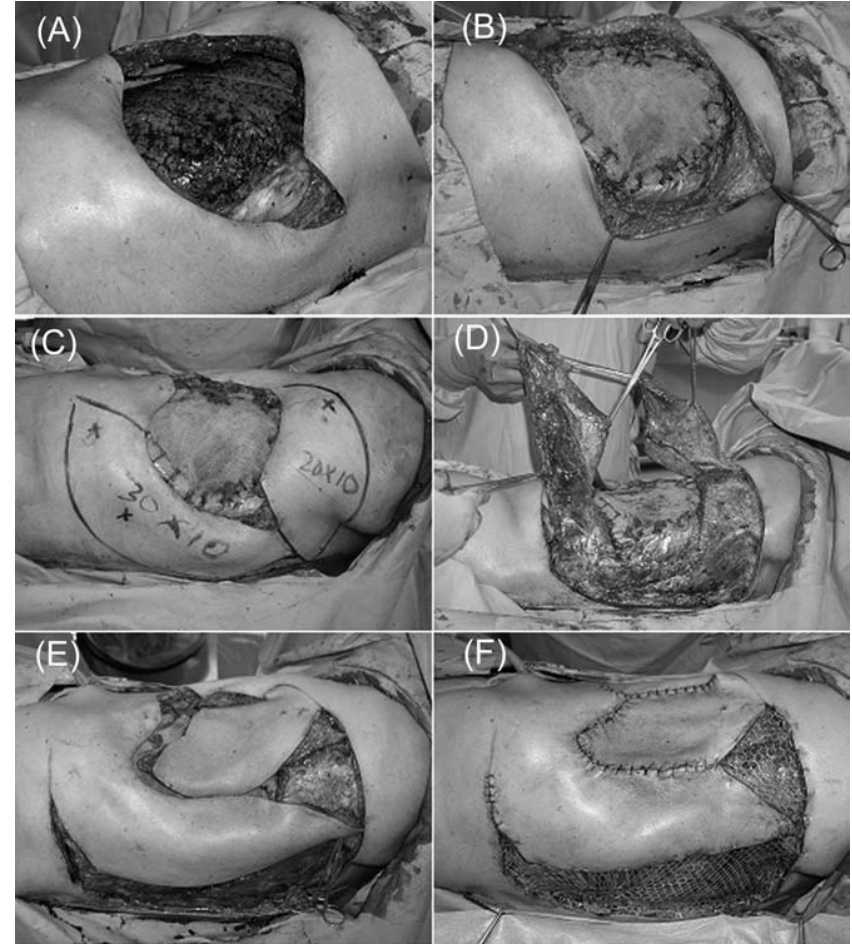

Figure 1. A, Chest wall defect. B, Skeletal reconstruction with Marlex mesh. C, Flap design: a horizontal scapular flap based on the circumflex scapular vessels and a flap based on the thoracodorsal perforators. D, Raised flaps. E, Flaps rotated to cover the defect. F, Final appearance.

patible with a desmoid tumor with a negative margin. At present, 5 years after the operation, the patient is well, without local recurrence and functional disabilities.

\section{Discussion}

Chest wall reconstruction has continued to grow, with delineation of new applications of flaps to repair increasingly complex defects, such as wide, full-thickness chest wall defects. Perforator flaps, a new modality, are based on the vessels that perforate muscles and distribute to the skin and subcutaneous tissue with a significant amount of blood flow that was composed exclusively of skin and subcutaneous fat. A single dominant perforator flap can cover almost the same wide area as myocutaneous flaps from the same regions. This is because the perforators are connected by numerous long, voluminous, subcutaneous anastomoses and by a closemeshed dermal network. ${ }^{4}$ This operation can be effectively used 


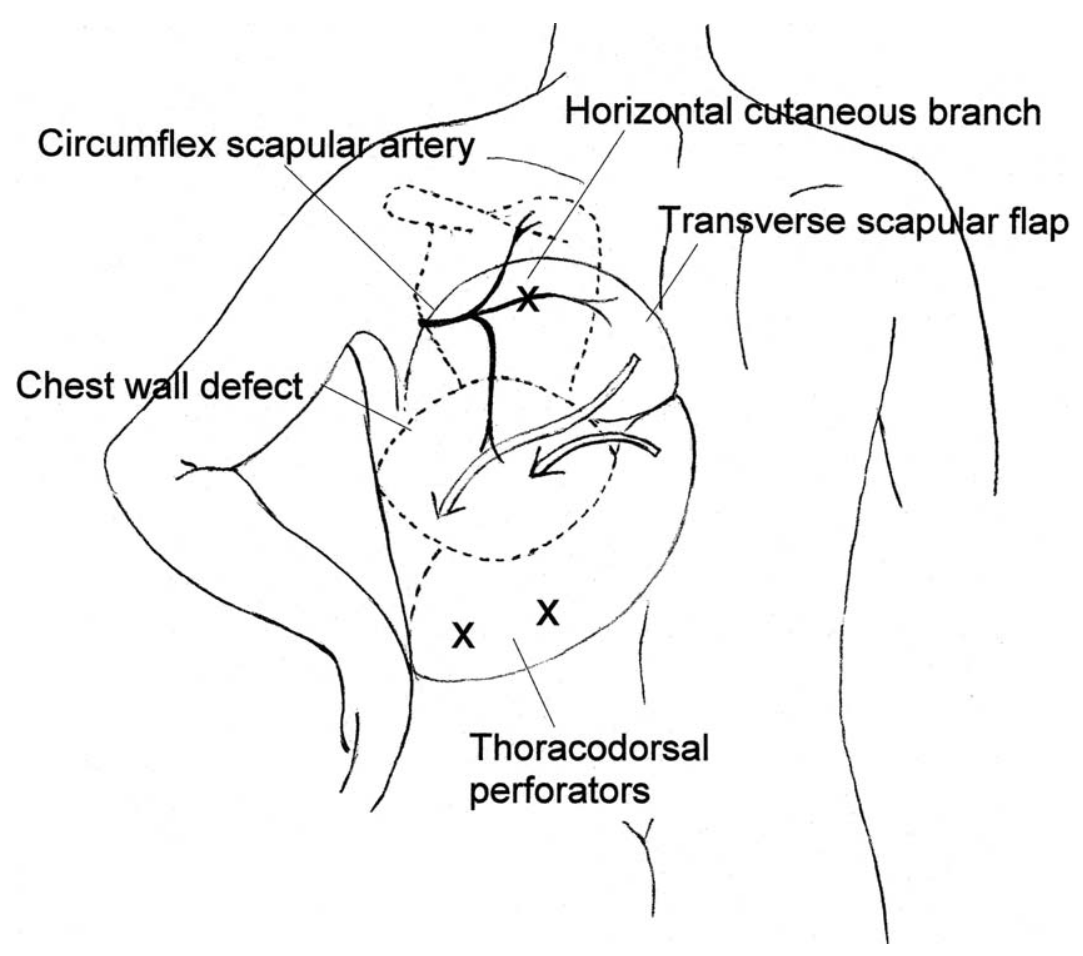

Figure 2. A schematic diagram of 2 perforator flaps.

for the reconstruction of the wide, full-thickness chest wall defect with Marlex mesh; this is a good alternative to the conventional myocutaneous flap. Advantages of perforator flaps over musculocutaneous flaps include (1) less donor site morbidity, (2) muscle sparing, and (3) improved postoperative recovery of the patient. ${ }^{5}$ The thickness of flaps was sufficient for the airtight reconstruction without deformity. The advantages of myocutaneous flaps include reliable vascular supply, large scope of reconstruction, and excellent bulk for filling soft-tissue defects withstanding infection or tension. On the other hand, the use of the flaps with the sacrifice of wide functional muscle units is occasionally accompanied by subsequent loss of donor site function or contour deformity. Although this newer operation with perforator flaps might require technical demands for flap design and dissection of perforators, as well as anatomic variation in the location and size of aimed perforators, perforator flaps are simpler and less invasive than myocutaneous flaps, with decreased need for analgesia and hospi- tal stay. ${ }^{5}$ To our knowledge, there has been no report on the use of perforator flaps instead of musculocutaneous flaps for wide chest wall reconstruction. The present case suggests the high potential of perforator flaps as a safe and effective option for chest wall reconstruction.

\section{References}

1. Koshima I, Soeda S. Inferior epigastric artery skin flaps without rectus abdominis muscle. Br J Plast Surg. 1989;42:645-8.

2. Kroll SS, Rosenfield L. perforator-based flaps for low posterior midline defects. Plast Reconstr Surg. 1988;81:561-6.

3. Ohsaki M, Maruyama Y. Anatomical investigations of the cutaneous branches of the circumflex scapular artery and their communications. Br J Plast Surg. 1993;46:160-3.

4. Taylor GI. The angiosomes of the body and their supply to perforator flaps. Clin Plast Surg. 2003;30:331-42.

5. Geddes CR, Morris SF, Neligan PC. Perforator flaps: evolution, classification, and applications. Ann Plast Surg. 2003;50:90-9. 